\title{
Mobile phone use and acoustic neuroma risk in Japan
}

\author{
T Takebayashi, S Akiba, Y Kikuchi, M Taki, K Wake, S Watanabe, N Yamaguchi
}

See end of article for authors' affiliations

\section{Correspondence to:} Professor $N$ Yamaguchi Department of Public Health, Tokyo Women's Medical University, 8-1 Kawadacho, Shinjuku-ku, Tokyo 162-8666, Japan: yamaguch@research. twmu.ac.jp

Accepted 4 August 2006 Published Online First 15 August 2006
Objectives: The rapid increase of mobile phone use has increased public concern about its possible health effects in Japan, where the mobile phone system is unique in the characteristics of its signal transmission. To examine the relation between mobile phone use and acoustic neuroma, a case-control study was initiated.

Methods: The study followed the common, core protocol of the international collaborative study, INTERPHONE. A prospective case recruitment was done in Japan for 2000-04. One hundred and one acoustic neuroma cases, who were 30-69 years of age and resided in the Tokyo area, and 339 age, sex, and residency matched controls were interviewed using a common computer assisted personal interview system. Education and marital status adjusted odds ratio was calculated with a conditional logistic regression analysis.

Results: Fifty one cases (52.6\%) and 192 controls (58.2\%) were regular mobile phone users on the reference date, which was set as one year before the diagnosis, and no significant increase of acoustic neuroma risk was observed, with the odds ratio (OR) being 0.73 (95\% $\mathrm{Cl} 0.43$ to 1.23). No exposure related increase in the risk of acoustic neuroma was observed when the cumulative length of use ( $<4$ years, 4-8 years, $>8$ years) or cumulative call time $(<300$ hours, $300-900$ hours, $>900$ hours) was used as an exposure index. The OR was $1.09(95 \% \mathrm{Cl} 0.58$ to 2.06$)$ when the reference date was set as five years before the diagnosis. Further, laterality of mobile phone use was not associated with tumours. Conclusions: These results suggest that there is no significant increase in the risk of acoustic neuroma in association with mobile phone use in Japan. he penetration of mobile telephony has been increasing in Japan as well as in other countries. In particular, the digital mobile phone system, first introduced into the Japanese market in 1993, gained popularity in only a few years. A remarkable change occurred in 1994 when handheld units began to be sold rather than rented. This triggered an explosive increase. According to the statistics for 2005, the number of mobile phone subscribers is approximately 91 million in Japan (Information and Communications in Japan, Ministry of Internal Affairs and Communications). The rapid increase of mobile phone use and the results of epidemiological studies reported from abroad in recent years have increased public concern about the possible health effects related to handheld cellular phone use. ${ }^{1-5}$ Among the various possible health effects, brain tumours have received the keenest public attention. Since electromagnetic field (EMF) exposure is relatively high only for the glial and meningial tissue close to the surface of the head, glioma and meningioma are the main concerns. ${ }^{6-8}$ Among head and neck tumours, acoustic neuroma is of particular concern due to the proximity of the tumour location to the antenna area, where the exposure to the EMF emitted from mobile phones is highest. $^{79}$

In light of need for further confirming evidence, the International Agency for Research on Cancer explored the possibility of conducting a large scale epidemiological study in 1997, and in 2000 began to coordinate an international collaborative case-control study on mobile phone use and brain tumours (the INTERPHONE study). Thirteen countries participated in this study, including Japan. ${ }^{10}$ Our study followed the common, core protocol of the INTERPHONE study, but the age range was wider in order to include more subjects. A questionnaire on habitual alcohol drinking and some nutritional indices, which were not included in the
INTERPHONE study protocol, were also added. Thus, we decided that the Japanese study was worth reporting separately. Furthermore, the mobile telephone system in Japan is unique in terms of the characteristics of the signal. The analogue system (first generation) of mobile phones was introduced in 1979. This system is no longer in use in Japan nor in many other countries. Of the second generation systems, the Personal Digital Cellular system prevailed from 1993 until quite recently. It is a Time Division Multiple Access system like the Global System for Mobile Communications (GSM) but it has a different waveform with a $50 \mathrm{~Hz}$ frame frequency $(217 \mathrm{~Hz}$ in GSM) and 3-slot communications in a frame (8-slots in GSM). A Code Division Multiple Access system, with the same specifications as the IS-95 system in the US, was also introduced in part in 1998. IMT-2000 (third generation) services began in 2001, and their market penetration is rapidly increasing. These differences between the situation in Japan and elsewhere suggest that a separate analysis for Japan is warranted.

\section{METHODS}

The study area was Tokyo, including 23 wards (the metropolitan area) and 14 cities (the municipal area) as well as 25 cities adjacent to Tokyo. According to a preliminary survey conducted before the case-control study, 30 out of 172 neurosurgery departments in Tokyo treated approximately $90 \%$ of brain tumour or acoustic neuroma cases in the area. Among those 30 departments, 21 brain surgery departments and one otorhinolaryngology department agreed to participate in the study. The case group consisted of hospitalised acoustic neuroma cases aged 30-69 years who were

Abbreviations: EMF, electromagnetic field; GSM, Global System for Mobile Communications 
diagnosed and treated in the participating departments. Case recruitment was done prospectively over the period from 1 December 2000 to 30 November 2004 at the participating departments. Of the 120 incident eligible acoustic neuroma cases, 101 cases $(84.2 \%)$ participated in the study. Thirteen could not be contacted by the study group, and six refused to participate. Cases treated surgically were verified histopathologically (code 9560/0 of ICD-O 3rd edition), but some were diagnosed by magnetic resonance imaging. Date of diagnosis was defined as the date when a neuroma was first identified radiologically.

Controls were selected from the general population using a random digit dialing method, in which phone numbers for fixed home phones were generated randomly. Controls were individually matched to the cases on age (five year category), sex, and residency. Controls were sought until at least two controls were found that corresponded to each case. For the acoustic neuroma study, $339(52.4 \%)$ controls were interviewed out of 647 control candidates who were identified as eligible. In addition, $136(21.0 \%)$ control candidates who did not agree to have a face-to-face interview agreed to respond to a brief survey, which included age, sex, brief history of mobile phone use, and some lifestyle factors, over the phone or by a self-administered paper questionnaire.

Each case and its matched controls were interviewed by the same interviewer. The interviewers were nurses or other health professionals, who were trained specifically for this study. A Japanese version of the computer assisted interview system developed for the INTERPHONE study was used for the face-to-face interviews. ${ }^{10}$ The interviews were conducted in the hospital for cases and at home or at the workplace for controls.

A subject was classified as a regular mobile phone user if he/she had used mobile phones for at least six months. Regular mobile phone users were asked about the start and stop dates for each mobile phone used, the average duration and frequency of calls, and other usage patterns in chronological order. Use of the Personal Handy-phone System (or PHS), which is a kind of cordless telephone system similar to Digital European Cordless Communication (or DECT) with a much lower power of emission than cellular telephones, was not included in the analysis presented in the Results section, while a separate analysis including PHS use showed similar results. Demographical variables, medical history, and occupational history were also recorded for all subjects. Clinical information regarding the cases was obtained from the participating departments.

After the interview was conducted, four cases were found to have been diagnosed before December 1999, more than one year before the study started. These four cases and their matched controls (nine controls) were excluded from the analysis. Thus, 97 cases and 330 matched controls were subject to analysis.

For the statistical analysis, the reference date for mobile phone use was set at one year before the date of diagnosis for each case to eliminate any effect of existing disease on recent mobile phone use, and the same date was applied for the matched controls. The selection of control candidates took 14 weeks on average, and thus the interviews of the controls were inevitably performed at later dates. The average time difference between the interviews of the cases and matched controls was 25 weeks. The use of the same reference date for both the cases and matched controls was therefore expected to avoid overestimation of mobile phone use for the controls.

For regular mobile phone use, two indices were considered: cumulative length of use and cumulative call time. The cumulative length of use was calculated by summing the length of use of each mobile phone. To calculate the length of use of the most recent mobile phone, either the reference date or the stop date of the last mobile phone-whichever came first-was adopted as the end of use date. The cumulative call time was the sum of call times (in hours) for all phones used. The call time for each phone was the product of the length of use (in days) and call duration (incoming and outgoing) per day. Call duration was calculated by multiplying the average length of one call by the number of calls per day.

A conditional logistic regression analysis was performed to estimate an odds ratio (OR). In the analysis, educational level (junior high school, high school or 2 year college, 4 year college or graduate school) and marital status (married, single, divorced, deceased) were adjusted for. The reference category for the calculation of OR was the non-user unless specified otherwise. To examine whether there was an association between the laterality of mobile phone use and the risk of tumour, we employed a conditional logistic regression analysis in which a subject was assumed to be exposed to EMF only when the self-reported side of mobile phone use was in agreement with his/her tumour laterality (ipsilateral use). For the controls, they were classified as exposed when the laterality of mobile phone use was the same as the laterality of the tumour of the matched case. If cases or controls answered that they could not specify the side of mobile phone use, the matched pair was excluded from the analysis. A similar analysis was done for contralateral use, in which a subject was assumed to be exposed when the self-reported side of mobile phone use was the opposite of the tumour laterality of the case. A control was classified as exposed, when the laterality of mobile phone use was the opposite of the laterality of the tumour of the matched case. If a subject answered that he/she used both ears for phone use, we assumed that the subject was "exposed" for both ipsilateral and contralateral analysis. The reference groups were defined as the non-regular user or regular user who used the opposite side of the tumour for ipsilateral use and as the non-regular user or regular user who used the same side of the tumour for contralateral use. These statistical analyses were done using STATA/SE version 8.2 (StataCorp, TX, USA). All statistical tests were two-sided. Further, a case-only analysis was done to examine the association between the laterality of the tumour and that of the phone use by the method proposed by Inskip et al. ${ }^{7}$ Briefly, the relative risk of a tumour with relation to side of phone use was estimated to be

$$
[(\sqrt{O R}+1) \div 2]
$$

where OR was calculated from the $2 \times 2$ table of side of phone use and the side of tumour, and the $\mathrm{p}$ value was obtained by Fisher's exact test (two-sided).

The study was approved by the institutional review boards of the participating institutes. In addition, the study protocol was reviewed by the Privacy Protection Subcommittee organised independently of the research group. The procedure during the study period was also monitored by this subcommittee, and no problem was identified with regard to privacy protection.

\section{RESULTS}

Among the 97 cases analysed, 80 (82.5\%) underwent surgical operation, seven $(7.2 \%)$ received gamma knife radiation therapy, and $10(10.3 \%)$ were not treated surgically or radiologically. Before the diagnosis, 92 (94.9\%) complained of subjective symptoms such as hearing difficulty, but five $(5.2 \%)$ were found to be incidentally without symptoms.

The basic characteristics of the cases and controls at the reference date are shown in table 1. There were no 


\begin{tabular}{|c|c|c|c|}
\hline & Control & Case & Total \\
\hline \multicolumn{4}{|l|}{ Age (years) } \\
\hline $30-39$ & $78(23.64)$ & $22(22.68)$ & $100(23.42)$ \\
\hline $40-49$ & 46 (13.94) & $14(14.43)$ & 60 (14.05) \\
\hline $50-59$ & $150(45.45)$ & $46(47.43)$ & $196(45.90)$ \\
\hline $60-69$ & 56 (16.97) & 15 (15.46) & 71 (16.63) \\
\hline \multicolumn{4}{|l|}{ Sex } \\
\hline Male & $132(40.00)$ & $45(46.39)$ & $177(41.45)$ \\
\hline Female & $198(60.00)$ & $52(53.61)$ & $250(58.55)$ \\
\hline \multicolumn{4}{|l|}{ Residential area } \\
\hline Tokyo (met*) & $124(37.58)$ & $42(43.30)$ & $166(38.88)$ \\
\hline Tokyo (munt) & $54(16.36)$ & 18 (18.56) & $72(16.86)$ \\
\hline Chiba & $42(12.73)$ & $10(10.31)$ & $52(12.18)$ \\
\hline Kanagawa & 48 (14.55) & $12(12.37)$ & 60 (14.05) \\
\hline Saitama & 62 (18.79) & $15(15.46)$ & 77 (18.03) \\
\hline \multicolumn{4}{|l|}{ Timing of interview } \\
\hline $00 / 12-01 / 11$ & 25 (7.58) & $20(20.62)$ & 45 (10.54) \\
\hline $01 / 12-02 / 11$ & 65 (19.69) & $32(32.99)$ & 97 (22.71) \\
\hline $02 / 12-03 / 11$ & $134(40.61)$ & 33 (34.02) & $167(39.11)$ \\
\hline $03 / 12-04 / 11$ & $99(30.00)$ & $12(12.37)$ & $111(26.00)$ \\
\hline $04 / 12-05 / 03$ & $7(2.12)$ & $0(0.00)$ & $7(1.64)$ \\
\hline \multicolumn{4}{|l|}{ Education } \\
\hline Junior high school & $18(5.45)$ & $9(9.28)$ & $27(6.32)$ \\
\hline High schools & $192(58.19)$ & 51 (52.57) & 243 (56.91) \\
\hline College/graduate & $120(36.36)$ & 37 (38.15) & $157(36.76)$ \\
\hline \multicolumn{4}{|l|}{ Marital status } \\
\hline Single & $26(7.88)$ & $14(14.43)$ & $40(9.37)$ \\
\hline Married & $280(84.85)$ & $79(81.44)$ & 359 (84.07) \\
\hline Divorced & $13(3.94)$ & $1(1.03)$ & 14 (3.28) \\
\hline Deceased & 11 (3.33) & $3(3.09)$ & $14(3.28)$ \\
\hline $\begin{array}{l}\text { * Metropolitan area of } T \\
\text { †Municipalities of Toky } \\
\text { fUp to } 9 \text { years. } \\
\text { \$Up to } 14 \text { years. } \\
\text {-16 years or more. }\end{array}$ & & & \\
\hline
\end{tabular}

substantial differences between the cases and controls in terms of age, sex, and residential area as well as educational level nor marital status. The latter two variables were selected a priori as confounding variables, and were expected to reflect socioeconomic status.

Among the cases, the mean age at the reference date was 47.4 (SD 9.6) years for mobile phone users, while that for non-users was 54.7 (SD 8.9) years. Similarly, for controls, the mean age was 48.4 (SD 9.8) years for mobile phone users and 55.6 (SD 9.8) years for non-users. As expected, mobile phone users tended to be younger than non-users, but the degree of this difference was quite similar between the cases and controls.

Table 2 summarises the risk estimates of mobile phone use. We reported an adjusted OR here, although the inclusion or exclusion of educational and marital status did not change the risk estimates substantially. No significant increase of acoustic neuroma risk was observed, with the OR being 0.73 with a 95\% confidence interval (CI) of 0.43 to 1.23 , when regular mobile phone use was compared between cases and controls. In addition, the OR was also estimated by setting the reference date as five years before the diagnosis of acoustic neuroma to further avoid the effects of existing disease. Nineteen cases (19.6\%) and 62 controls (18.8\%) had used mobile phones regularly, and OR was 1.09 (95\% CI 0.58 to 2.06) when the reference date was set at five years before the diagnosis.

When cumulative length of use was included in the conditional logistic regression model as a continuous variable, the OR for a one year increase in cumulative length of use was 0.998 ( $95 \%$ CI 0.991 to $1.006 ; \mathrm{p}=0.652$ ). Cumulative length of use was further categorised into four classes: nonuser $(0),>0$ to $<4$ years, 4 to $<8$ years, and $\geqslant 8$ years, because regular use of 10 years or more was found to be very rare-it was present in only one case $(1.0 \%)$ and eight controls $(2.4 \%)$. When the cumulative length of use as classified above was compared between cases and controls, no significant increasing trend in risk was observed $(p=0.636)$. ORs for $<4$ years, $<8$ years, and $\geqslant 8$ years were $0.70,0.76$, and 0.79 , respectively.

The OR for a 300 hour increase in cumulative call time was estimated to be 1.000 ( $95 \%$ CI 0.999 to $1.002 ; p=0.541$ ) when cumulative call time was included in the conditional logistic regression model as a continuous variable. When cumulative call time was further divided into four categories (non-user $(0),>0$ to $<300$ hours, 300 to $<900$ hours, and $\geqslant 900$ hours), no significant increasing trend was observed for the risk of acoustic neuroma in relation to increasing cumulative call time $(p=0.256)$. ORs for $<300$ hours, $<900$ hours and $\geqslant 900$ hours were 0.67, 1.37, and 0.67, respectively.

All the mobile phones used by the cases and controls were classified as analogue or digital type according to the make of phones, and the risk of acoustic neuroma was estimated for each type. Since there was no subject who used only analogue type phones, the type of phone was classified in one of these two categories: both analogue and digital or digital only. The OR for both analogue and digital was 1.19 (95\% CI 0.37 to 3.79 ) and that for digital only was 0.68 (95\% CI 0.40 to 1.18 ).

With regard to the laterality of mobile phone use and the laterality of tumour location, no significant association was found when ipsilateral use was assumed. OR was 0.90 (95\% CI 0.50 to 1.62 ). Also, no association was found when contralateral use was assumed. OR was 0.93 (95\% CI 0.55 to 1.59). Laterality was further examined by the method proposed by Inskip et $a^{7}$ with a case-only design (table 3 ). The relative risk of developing acoustic neuroma on the same side as the side of regular mobile phone use was 0.72 $(\mathrm{p}=0.01)$. 
Table 2 Case-control comparisons of indices for mobile phone use

\begin{tabular}{|c|c|c|c|c|}
\hline & Control & Case & $p$ for trend & Odds ratio* $95 \% \mathrm{Cl}$ ) \\
\hline \multicolumn{5}{|l|}{ Mobile phone use } \\
\hline Non-user & $138(41.82)$ & $46(47.42)$ & & 1.0 \\
\hline Regular user & $192(58.18)$ & $51(52.58)$ & & $0.73(0.43-1.23)$ \\
\hline \multicolumn{5}{|c|}{ Cumulative length of use (years) } \\
\hline Non-user & $138(41.82)$ & $46(47.42)$ & & 1.0 \\
\hline$<4$ & 111 (33.64) & $26(26.80)$ & & $0.70(0.39-1.27)$ \\
\hline $4-7$ & $69(20.91)$ & $21(21.65)$ & & $0.76(0.38-1.53)$ \\
\hline $8+$ & $12(3.64)$ & $4(4.12)$ & $p=0.702$ & $0.79(0.24-2.65)$ \\
\hline \multicolumn{5}{|c|}{ Cumulative call time (hours) } \\
\hline Non-user & $138(41.82)$ & $46(47.42)$ & & 1.0 \\
\hline$<300$ & $147(44.55)$ & 35 (36.08) & & $0.67(0.38-1.17)$ \\
\hline $300-900$ & $17(5.15)$ & $9(9.28)$ & & $1.37(0.54-3.50)$ \\
\hline $900+$ & $28(8.48)$ & 7 (7.22) & $p=0.694$ & $0.67(0.25-1.83)$ \\
\hline \multicolumn{5}{|c|}{ Type of mobile phone used } \\
\hline Non-user & $138(41.82)$ & $46(47.42)$ & & 1.0 \\
\hline Analogue+digital & $10(3.03)$ & $5(5.15)$ & & $1.19(0.37-3.79)$ \\
\hline Digital only & $182(54.15)$ & $46(47.42)$ & & $0.68(0.40-1.18)$ \\
\hline \multirow{2}{*}{\multicolumn{5}{|c|}{$\begin{array}{l}\text { Laterality of tumour location } v \text { mobile phone use } † \\
\text { Ipsilateral use }\end{array}$}} \\
\hline & & & & \\
\hline Reference & $255(77.74)$ & $\begin{array}{l}76(79.17) \\
20(2083)\end{array}$ & & 1.0 \\
\hline \multicolumn{5}{|l|}{ Contralateral use§ } \\
\hline Reference & $199(60.67)$ & $59(61.46)$ & & 1.0 \\
\hline Contra-use & $129(39.33)$ & $37(38.54)$ & & $0.93(0.55-1.59)$ \\
\hline
\end{tabular}

*Adjusted for education and marital status.

†One case was excluded from the analysis because the laterality of mobile phone use was unknown. For the analysis, the matched pair was excluded from the analysis.

flpsilateral use: a case was classified as exposed when the laterality of mobile phone use was the same as the laterality of tumour. A control was classified as exposed when the laterality of mobile phone use was the same as the laterality of the tumour of the matched case. If a subject used both ears for phone use, the subject was classified as exposed. The reference group was defined as the non-regular users or regular users whose phone use occurred on the side opposite the tumour.

$\S$ Contralateral use: a case was classified as exposed when the laterality of mobile phone use was the opposite of the laterality of the tumour. A control was classified as exposed when the laterality of mobile phone use was the opposite of the laterality of the tumour of the matched case. If a subject used both ears for phone use, the subject was classified as exposed. The reference group was defined as the non-regular users or regular users whose phone use occurred on the same side as the tumour.

\section{DISCUSSION}

The present study did not show any increased risk of acoustic neuroma in association with mobile phone use in Japan. No increasing tendency was observed for acoustic neuroma risk in association with increasing cumulative length of use, nor with increasing cumulative call time. The small nonsignificant decrease in risk for three categories of cumulative length of use $(0.70,0.76$, and 0.79$)$ could probably be explained by the effect of existing latent disease, as will be discussed later. The OR for the 300-600 hour category of cumulative call time showed a non-significant increase of 1.37, but this increase could be attributed to statistical uncertainty, as indicated by the wide confidence interval. When the mobile phones were classified into analogue and digital types, negative results were consistently observed for those who had used both analogue and digital mobile phones as well as for those who had used only digital phones. This finding further supports the hypothesis that mobile phonesboth analogue and digital-did not increase the risk of acoustic neuroma.

Thus far, five epidemiological studies on the national level have been reported regarding mobile phone use and acoustic

Table 3 Case-only analysis: tumour side and mobile phone use

\begin{tabular}{|c|c|c|c|c|c|}
\hline \multirow[b]{2}{*}{ Tumour side } & \multicolumn{4}{|c|}{ Regular side of mobile phone use } & \multirow[b]{2}{*}{ Total } \\
\hline & Right & Left & Both & Don't know & \\
\hline Right & 5 & 12 & 4 & 1 & 22 \\
\hline Left & 18 & 8 & 1 & 0 & 27 \\
\hline Both & 0 & 0 & 2 & 0 & 2 \\
\hline Total & 23 & 20 & 7 & 1 & 51 \\
\hline
\end{tabular}

neuroma; three studies were conducted before the INTERPHONE study was initiated, ${ }^{679}{ }^{11}$ and the remaining two studies were a part of the INTERPHONE study. ${ }^{12}{ }^{13}$ In a case-control study of brain tumours in the United States, 96 acoustic neuroma cases diagnosed between 1994 and 1998 were compared with frequency matched controls, and no increase in risk was found in association with mobile phone use, duration of use, or frequency of use. ${ }^{7}$ Another casecontrol study conducted in the United States also reported negative results. ${ }^{9}$ In this case-control study, 90 acoustic neuroma cases were compared with hospital controls. The OR was estimated to be 0.9 , and no risk was found to be associated with the frequency, duration, or lifetime hours of use. In a case-control study of brain tumours conducted in Sweden, 148 acoustic neuroma cases were compared with population based matched controls, and an increased risk of acoustic neuroma was found in relation to the use of analogue phones; the OR was 4.4 with $95 \%$ CI 2.1 to $9.2 .^{11}$ Curiously, however, the ORs for ipsilateral use (that is, mobile phone use on the same side as the tumour location) and for contralateral use (mobile phone use on the opposite side of the tumour location) showed similar increases in risk; the OR for ipsilateral use was 4.2 (95\% CI 1.6 to 11$)$, whereas that for contralateral use was 3.7 (95\% CI 1.4 to 9.8).

Two case-control studies that were part of the INTERPHONE study-one conducted in Denmark and the other in Sweden-reported inconsistent results. In the casecontrol study in Denmark, 106 acoustic neuroma cases were compared with 212 matched controls, and no increase in risk was found in association with the use of mobile phones. No increase in risk was identified for mobile phone use of 10 years or more, as compared with short term use. Further, no difference was found based on the laterality of mobile phone use and tumour location. ${ }^{12}$ In the case-control study in Sweden, however, an increased risk was found in relation to 
the use of mobile phones for 10 years or more $(\mathrm{OR}=1.9,95 \%$ CI 0.9 to 1.5), and an even higher increase in risk was found when the phone use considered was restricted to the same side as the tumour ( $\mathrm{OR}=3.9,95 \%$ CI 1.6 to 9.5). However, the overall risk for mobile phone use did not show any increase, with the OR being 1.0. ${ }^{13}$

Recently, a pooled case-control analysis of 678 cases and 3553 controls from case-control studies in Sweden, Denmark, Norway, Finland, and the United Kingdom (two study sites) was reported. ${ }^{14}$ All studies were conducted as part of the INTERPHONE study. No increase in risk was identified in association with mobile phone use $(\mathrm{OR}=0.9)$. No increased risk was found in relation to the duration of use, lifetime cumulative hours of use, or the number of mobile phone calls made. An increase in risk was found, however, for ipsilateral use of mobile phones for 10 years or more $(\mathrm{OR}=1.8,95 \% \mathrm{CI}$ 1.1 to 3.1. This result should be interpreted with caution, however, because more than one third of such cases were from the Swedish study. Thus, the results that have been reported so far are inconsistent. Three studies reported negative results, ${ }^{7}{ }^{12}$ whereas two reported increased risk. ${ }^{11}{ }^{13}$

Such inconsistencies are generally explained by differences in study design and in exposure characteristics, in addition to statistical uncertainty. With regard to the study design, the potential effect of existing latent disease should be taken into account. The OR for regular use was rather lower than unity (0.73), though the difference was not significant. This means that acoustic neuroma cases tended to not use mobile phones when compared with controls. The reference date for the use of mobile phones was set at one year before the diagnosis of the tumour, so that the effect of existing latent disease on the use of mobile phones did not distort the risk estimation. The lower OR can be explained as a statistical uncertainty, but it might also suggest that some of the cases might have been affected, even one year before diagnosis, by symptoms of latent disease. The fact that $95 \%$ of the cases reported having symptoms before diagnosis and significant decrease of the risk estimate of developing a tumour on the side of phone use in the case-only analysis support this possibility. The numbers of mobile phone users at one and five years before diagnosis were compared so as to estimate the fraction of the cases and controls who started using mobile phones between one and five years before diagnosis. Among the cases, $33.0 \%$ were found to have started using mobile phones during that period, whereas $39.4 \%$ of controls did so during the same period. This also suggests that latent disease affected the use of mobile phones among the cases. This possible bias can be called "latent disease bias", which would affect the risk estimate downward for recent use. It should also be mentioned, however, that, even when we excluded the use of mobile phones five years before diagnosis, the observed OR did not show any increase in risk.

With regard to the exposure characteristics, two factors are worth considering: one is that the type of mobile phone, such as analogue or digital, and the type of signal transmission mode differ by country; the other is the use patterns of mobile phones, such as the duration and frequency of use. In particular, the difference between the analogue and digital types of mobile phone is of special interest, because the two studies in Sweden ${ }^{11}{ }^{13}$ reported increased risk among analogue phone users, whereas the two studies in Denmark and Japan did not find any increased risk for analogue phone users. The two US studies did not analyse this difference. ${ }^{79}$ The most evident association was reported by Hardel et al. ${ }^{11}$ The OR of acoustic neuroma was evidently increased among analogue type mobile phone users but was only slightly increased among digital mobile phone users. However, the laterality of the tumours was not related to the OR increase in their study; the increase was observed both in ipsilateral and contralateral use. Thus it seems unlikely that the observed increase in risk for analogue phone use was caused by the exposure to EMF from the phone. The study by Lonn et al did show a stronger increase in risk for ipsilateral use, but it was not restricted to analogue phones. ${ }^{13}$

Since a case-control study relies on inquiring into past exposure on the basis of the subjects' recall, the risk estimate should be carefully examined for possible biases that might distort it. In particular, the risk estimate of mobile phone use and acoustic neuroma is vulnerable to biases because the disease is closely related to hearing problems on the affected side. $^{15}$

In addition to the effect of the aforementioned "existing latent disease" on the risk estimate, several other factors are worth considering in interpreting the results. The recall bias is another potentially serious source of bias in a case-control study. In general, cases tend to make more effort than controls to recall their experiences in the past, as they think these experiences might be related to their disease. The difference in recall between cases and controls tends to be larger for events in the remote past than for recent events. The present case-control study was carefully designed to avoid such recall bias, but there is, of course, no guarantee of the absence of recall bias, especially for mobile phone use occurring a long time before the diagnosis. In the present study, however, no increase in risk was observed in relation to mobile phone use a long time before the diagnosis. As already discussed, mobile phones rapidly became popular around 1994 in Japan, and there are very few cases or controls who used mobile phones more than 10 years ago.

Another potential recall bias, which seems unique to the case-control study of mobile phone use and acoustic neuroma, is related to the laterality of mobile phone use and tumour location. It is possible that a case subject may recall whether he/she used mobile phones more frequently on the side of the head that was affected by the disease than on the other side. The results of ipsilateral and contralateral analysis, however, showed no increase in risk associated with mobile phone use on the side of the head affected by the disease.

Taking account of the slow growing nature of this benign tumour, we need to consider the possibility of detection bias at work. Symptoms such as tinnitus and hearing difficulty often trigger a diagnosis of acoustic neuroma, and the use of mobile phones might increase the likelihood of finding such symptoms, and might thus lead to earlier diagnosis of the disease among mobile phone users. If this detection bias is at work, an increase in OR would be expected due to a higher detection rate of disease among mobile phone users compared with non-users. We compared the age at tumour diagnosis of mobile phone users and that of non-users in order to examine this possibility. The difference between cases and controls was small; 7.3 years for cases and 7.2 years for controls, indicating that a detection bias did not distort the results in our study.

As our study depended on the voluntary participation of cases and controls, the possibility of selection bias should also be considered. ${ }^{16}$ The selection bias, which could have lowered the observed OR, could have resulted in a lower participation rate of mobile phone users among cases and a higher participation rate of users among controls. Among cases, there seems to be little possibility, if any, that users had a lower participation rate. Contrarily, efforts were made to avoid a higher participation rate of users by masking the major purpose of our study, because knowing the study purpose could raise mobile phone users' interest in the study, leading to a higher participation rate. Even so, we cannot totally discount the possibility that the participation rate was higher among those who were interested in the potential 


\section{Main messages}

- The current prevalence of mobile phone use in Japan is over $50 \%$, in both males and females.

- Any increased risk of acoustic neuroma was not shown in association with regular mobile phone use.

- Risk of acoustic neuroma did not increase with the duration of mobile phone use, nor with the total call time.

- No increased risk was observed even when mobile phone use in recent years was not accounted for.

\section{Policy implications}

- There is no significant increase in the risk of acoustic neuroma in association with mobile phone use in Japan.

- Future studies should focus on long term users with more than 10 years of use and on those who have used analogue phones in the past.

health hazards of mobile phone use. Also, it is not likely that mobile phone users had a higher participation rate among controls. A differential participation rate could have been introduced in our study in the phase of recruitment by random digit dialing, and also in the phase of soliciting to participate in the study. In the random-digit dialing procedure, efforts were made to avoid missing individuals who were away from home for a greater number of hours. We assumed that mobile phone users tended to be more active outside of the home, and missing those who were more active would result in a smaller number of mobile phone users in the control group. Also, in the procedure through which participation was solicited by telephone contact, efforts were made to avoid missing those with busier lives, because we assumed that mobile phone users tended to be busier and that the participation rate of users would be lower than it should have been unless we made efforts not to miss such users. In fact, the proportion of regular mobile phone users among the control candidates who did not participate in the full interview but agreed to respond to a brief questionnaire (21\% of eligible controls) was comparable with that for the controls who participated in the full, face-to-face interview (52.4\% of eligible controls). Overall, it seems that selection bias was less likely to be at work in the process of case and control selection. It should be mentioned that the higher participation rate among cases than controls may lower the OR to some extent, since Lahkola et al suggested that mobile phone users were more often among controls who fully participated than those who completely refused to. ${ }^{16}$ Unfortunately however, we could not compare these figures with completely-refused controls.

In summary, the five studies reported thus far and the present study have not shown any consistent increased risk of acoustic neuroma in association with mobile phone use. In this study, the risk of acoustic neuroma does not increase with the duration of mobile phone use, nor with the total call time. Future studies should focus on long term users with more than 10 years of use and on those who have used analogue phones in the past. Case-only studies based on the analysis method proposed by Inskip et al would also be valuable.

\section{ACKNOWLEDGEMENTS}

The study conducted in Japan was fully funded by the Ministry of Internal Affairs and Communications of Japan. We are grateful for the cooperation of the surgeons and staff of the participating institutions.

\section{Authors' affiliations}

T Takebayashi, Y Kikuchi, Department of Preventive Medicine and Public Health, Keio University School of Medicine, Tokyo, Japan S Akiba, Department of Epidemiology and Preventive Medicine, Kagoshima University Graduate School of Medical and Dental Sciences, Kagoshima, Japan

M Taki, Department of Electrical and Electronic Engineering, Tokyo Metropolitan University, Tokyo, Japan

K Wake, S Watanabe, Electromagnetic Compatibility Group, Applied Electromagnetic Research Center, National Institute of Information and Communications Technology, Tokyo, Japan

N Yamaguchi, Department of Public Health, Tokyo Women's Medical University, Tokyo, Japan

Competing interests: none.

\section{REFERENCES}

1 Rothman KJ, Chou CK, Morgan R, et al. Assessment of cellular telephone and other radio frequency exposure for epidemiologic research. Epidemiology 1996; 7:291-8

2 Violanti JM, Marshall JR. Cellular phones and traffic accidents: an epidemiological approach. Accid Anal Prev 1996;28:265-70.

3 Repacholi MH. Low-level exposure to radiofrequency electromagnetic fields: health effects and research needs. Bioelectromagnetics 1998;19:1-19.

4 Blettner M, Berg G. Are mobile phones harmful? Acta Oncol 2000;39:927-30.

5 Rothman KJ. Epidemiological evidence on health risks of cellular telephones. Lancet 2000;356:1837-40.

6 Muscat JE, Malkin MG, Thompson S, et al. Handheld cellular telephone use and risk of brain cancer. JAMA 2000;284:3001-7.

7 Inskip PD, Tarone RE, Hatch EE, et al. Cellular-telephone use and brain tumors. N Engl J Med 2001;344:79-86.

8 Johansen C, Boice J Jr, McLaughlin J, et al. Cellular telephones and cancer-a nationwide cohort study in Denmark. J Natl Cancer Inst 2001;93:203-7.

9 Muscat JE, Malkin MG, Shore RE, et al. Handheld cellular telephones and risk of acoustic neuroma. Neurology 2002;58:1304-6.

10 Cardis E, Kilkenny M. International case-control study of adult brain, head and neck tumours: results of the feasibility study. Radiat Prot Dosimetry 1999:83:179-83.

11 Hardell L, Mild KH, Carlberg M. Further aspects on cellular and cordless telephones and brain tumours. Int J Oncol 2003;22:399-407.

12 Christensen HC, Schuz J, Kosteljanetz M, et al. Cellular telephone use and risk of acoustic neuroma. Am J Epidemiol 2004;159:277-83.

13 Lonn S, Ahlbom A, Hall P, et al. Mobile phone use and the risk of acoustic neuroma. Epidemiology 2004;15:653-9.

14 Schoemaker MJ, Swerdlow AJ, Ahlbom A, et al. Mobile phone use and risk of acoustic neuroma: results of the Interphone case-control study in five North European countries. Br J Cancer 2005;93:842-8.

15 Savitz DA. Mixed signals on cell phones and cancer. Epidemiology 2004;15:651-2.

16 Lahkola A, Salminen T, Auvinen A. Selection bias due to differential participation in a case-control study of mobile phone use and brain tumors. Ann Epidemiol 2005;15:321-5. 